\title{
Theoretical description of hydrogen bonding in oxalic acid dimer and trimer based on the combined extended-transition-state energy decomposition analysis and natural orbitals for chemical valence (ETS-NOCV)
}

\author{
Mariusz P. Mitoraj • Rafal Kurczab • Marek Boczar • \\ Artur Michalak \\ Received: 15 December 2009 / Accepted: 3 May 2010/Published online: 28 May 2010 \\ (C) The Author(s) 2010. This article is published with open access at Springerlink.com
}

\begin{abstract}
In the present study we have analyzed hydrogen bonding in dimer and trimer of oxalic acid, based on a recently proposed charge and energy decomposition scheme (ETS-NOCV). In the case of a dimer, two conformations, $\alpha$ and $\beta$, were considered. The deformation density contributions originating from NOCV's revealed that the formation of hydrogen bonding is associated with the electronic charge deformation in both the $\sigma-\left(\Delta \rho_{\sigma}\right)$ and $\pi$-networks $\left(\Delta \rho_{\pi}\right)$. It was demonstrated that $\sigma$-donation is realized by electron transfer from the lone pair of oxygen on one monomer into the empty $\rho_{H-O}^{*}$ orbital of the second oxalic acid fragment. In addition, a covalent contribution is observed by the density transfer from hydrogen of H-O group in one oxalic acid monomer to the oxygen atom of the second fragment. The resonance assisted component $\left(\Delta \rho_{\pi}\right)$, is based on the transfer of electron density from the $\pi$-orbital localized on the oxygen of $\mathrm{OH}$ on one oxalic acid monomer to the oxygen atom of the other fragment.
\end{abstract}

M. P. Mitoraj · A. Michalak $(\bowtie)$

Department of Theoretical Chemistry, Faculty of Chemistry,

Jagiellonian University,

R.Ingardena 3,

30-060 Cracow, Poland

e-mail: michalak@chemia.uj.edu.pl

R. Kurczab · M. Boczar

Department of Physical Chemistry, Faculty of Chemistry,

Jagiellonian University,

R.Ingardena 3,

30-060 Cracow, Poland

R. Kurczab

Department of Medicinal Chemistry, Institute of Pharmacology,

Polish Academy of Sciences,

12 Smętna Street,

31-343 Cracow, Poland
ETS-NOCV allowed to conclude that the $\sigma(\mathrm{O}-\mathrm{HO})$ component is roughly eight times as important as $\pi$ (RAHB) contribution in terms of energetic estimation. The electrostatic factor $\left(\Delta \mathrm{E}_{\text {elstat }}\right)$ is equally as important as orbital interaction term $\left(\Delta \mathrm{E}_{\text {orb }}\right)$. Finally, comparing $\beta$ dimer of oxalic acid with trimer we found practically no difference concerning each of the O--HO bonds, neither qualitative nor quantitative.

Keywords ETS-NOCV. Extended transition state. Natural orbitals for chemical valence - Oxalic acid dimer and trimer $\cdot$ Resonance assisted hydrogen bonds

\section{Introduction}

Hydrogen bonding (HB) plays an important role in biochemistry $[1,2]$. Accordingly, many attempts were made to describe HB-phenomenon in both experimental and theoretical laboratories [3-11]. For the purpose of understanding the nature of hydrogen bonding, theoretical approaches appeared to be of significant importance [1219]. Especially, electrostatic-covalent HB model, derived by Gilli $[20,21]$ from the systematic analysis of structural and spectroscopic data, allowed to make a comprehensive classification of $\mathrm{H}$-bonds in a different chemical classes: $e$. $g$., charge assisted hydrogen bonds, low barrier hydrogen bonds, dihydrogen bonds, and resonance-assisted hydrogen bonds (RAHBs). It clearly shows that different factors can influence the nature of hydrogen connections.

For the purpose of HB description, various energy decomposition analysis (EDA) were also used [22-25, 46]. In such decomposition schemes, the total interaction energy between interacting sub-systems is divided up into a few 
chemically meaningful contributions (electrostatic, exchange, charge-transfer, relaxation, etc.). Despite great usefulness of these methods, as they allow to extract stabilizing and destabilizing factors, none of them provide direct, qualitative and quantitative, separated information about $\sigma$-and $\pi$-contributions. Such specific knowledge on the hydrogen bonding phenomenon can be useful in understanding the structures of bio-molecules and further in a description of reactivity.

We have recently developed the ETS-NOCV scheme by combining the extended transition state (ETS) [26, 27] energy decomposition approach with the natural orbitals for chemical valence (NOCV) density analysis method [2834]. It was shown that ETS-NOCV is able to extract and directly quantify the crucial components $(\sigma, \pi, \delta$, etc. $)$ that participate in various types of chemical bonds including predominantly donor-acceptor and covalent interactions $[35,36]$. Furthermore, we have shown that ETS-NOCV can also be useful in a description of weak bonds [35, 36], including intra - and inter-molecular hydrogen bonding. For example, we have found, based on the analysis of intermolecular bonding in Adenine-Thymine base pair, that ETS-NOCV provides separated, qualitative and quantitative information not only about both $\sigma(\mathrm{O}-\mathrm{HN})$ and $\sigma(\mathrm{N}-\mathrm{HN})$ interactions, but also about the resonance assisted hydrogen bonding contribution $(\pi)$ [35].

The main goal of this article is to extend our study on weak intermolecular interaction by characterization of hydrogen bonding in the dimeric and trimeric systems consisting of both $\sigma(\mathrm{O}-\mathrm{HO})$ and $\pi$-interactions between the monomers. We will consider in our analysis the oxalic acid dimers exhibiting two different conformations $\alpha$ and $\beta$ (1 and 2), Fig. 1. In addition, we will investigate oxalic acid trimers, 3 and 4, based on two different bond-partitioning, as indicated in Fig. 1. The purpose of doing so is to obtain a compact description of each $\mathrm{O}-\mathrm{-HO}$ interaction in trimer of oxalic acid. To the best of our knowledge this is the first study reporting on the character of bonding in the oxalic acid systems based on charge and energy decomposition analysis. All structures discussed here were fully optimized after initial guesses taken from the Cambridge Structural Database $[37,38]$. For the sake of clarity of presentation we will use throughout our study the " $\alpha$-dimer" and " $\beta$-dimer" terms to describe the oxalic acid dimers that exhibit two different conformations $\alpha$ and $\beta$, respectively.

\section{Computational details}

All the calculations, within dDensity functional theory approach, were based on the Amsterdam density functional (ADF2009) program in which ETS-NOCV scheme was implemented [39-43]. The Becke-Perdew exchange- correlation functional $[44,45]$ was applied (BP86). A standard triple-zeta STO basis containing two sets of polarization functions was adopted for all of the elements (TZ2P). Auxiliary $s, p, d, f$, and $g$ STO functions, centered on all nuclei, were used to fit the electron density. It was proven previously for similar systems $[35,46]$ that optimized structures and interaction energies based on BP86/TZ2P calculations are in line with the corresponding estimates obtained from the experiment and from high-level $a b$ initio methods. The contours of deformation densities were plotted using ADF-GUI [47].

In our analysis each of the system is divided up into two individual fragments A and B, as shown schematically by a thick line in Fig. 1. Then we used the ETS-NOCV method to study the interaction between A and B, as they are brought together to form $\mathrm{A} \cdots \mathrm{B}$. Thus, our analysis is based on the bonding between the two close shell molecular fragments.

\section{Computational methods}

As we mentioned in the previous paragraph, our analysis is based on the ETS-NOCV approach which is a combination of the extended transition state (ETS) $[26,27]$ method with the natural orbitals for chemical valence (NOCV) [28-34] scheme.

We shall start by presenting the basic concepts of the ETS method. In this scheme, the total bonding energy between the interacting fragments $\left(\Delta \mathrm{E}_{\text {total }}\right)$ is divided into four chemically meaningful components Eq. 1:

$\Delta \mathrm{E}_{\text {int }}=\Delta \mathrm{E}_{\text {dist }}+\Delta \mathrm{E}_{\text {elstat }}+\Delta \mathrm{E}_{\text {Pauli }}+\Delta \mathrm{E}_{\text {orb }}$.

The first component, referred to as the distortion term $\Delta \mathrm{E}_{\text {dist }}$, represents the amount of energy required to promote the separated fragments from their equilibrium geometry to the structure they will take up in the combined molecule. The second term, $\Delta \mathrm{E}_{\text {elstat }}$, corresponds to the classical electrostatic interaction between the promoted fragments as they are brought to their positions in the final complex. The third term, $\Delta \mathrm{E}_{\text {Pauli }}$, accounts for the repulsive Pauli interaction between occupied orbitals on the two fragments in the combined complex. Finally, the last term, $\Delta \mathrm{E}_{\text {orb }}$, represents the stabilizing interactions between the occupied molecular orbitals on one fragment with the unoccupied molecular orbitals of the other fragment as well as the mixing of occupied and virtual orbitals within the same fragment (intra-fragment polarization) after the two fragments have been united. We can write the change in density that gives rise to $\Delta \mathrm{E}_{\text {orb }}$ as

$\Delta \rho(1)=\sum_{\lambda} \sum_{v} \Delta P_{\mu \nu} \lambda(1) v(1)$ 
Fig. 1 The hydrogen bonded systems studied in the present work (1-4). A thick line represents the way of fragmentation used in a description of hydrogen bonding. In bonding analysis of 4 the middle monomer was treated as first fragment (a) and the terminal monomers as the second fragment (b)
1

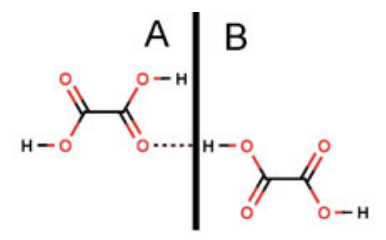

2
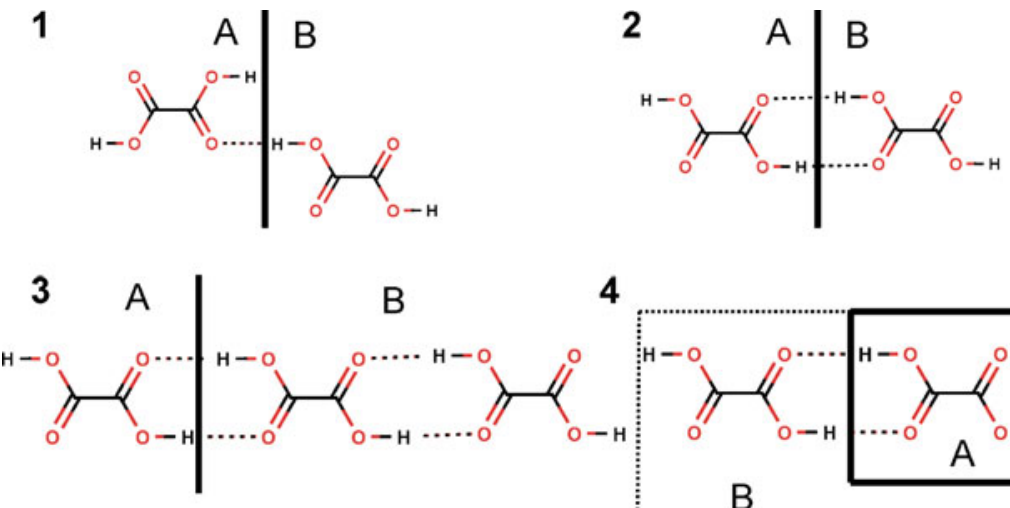

4

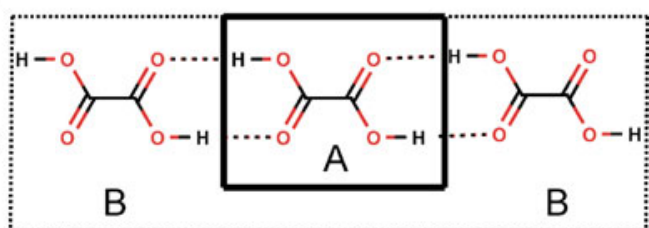

where the sum is over all the occupied and virtual molecular orbitals on the two fragments. It now follows from the ETS scheme that the $\Delta \mathrm{E}_{\text {orb }}$ term is given by $[26,27]$

$\Delta E_{\text {orb }}=\sum_{\lambda} \sum_{\mu} \Delta P_{\lambda \mu} F_{\lambda \mu}^{T}$

where $F_{\lambda \mu}^{T S}$ is a Kohn-Sham Fock matrix element that is defined in terms of a (transition state) potential that is mid between that of the combined fragments and the final molecule. It should be emphasized that the meaning of "transition state" in the ETS approach is completely different from that commonly used in the context of the transition state theory.

Turning next to the NOCV approach, we note that historically the natural orbitals for chemical valence (NOCV) [28-34] have been derived from the NalewajskiMrozek valence theory[48-54]. However, from a mathematical point of view the NOCV's, $\psi_{\mathrm{i}}$, are simply defined as the eigenvectors,

$\psi_{i}(1)=\sum_{\lambda}^{M} C_{i, \lambda} \lambda(1)$

that diagonalize the deformation density matrix $\Delta P$ introduced in Eq. 2. Thus,

$\Delta P C_{i}=v_{i} C_{i} \quad ; \quad i=1, M$

where $M$ denotes the total number of molecular orbitals on the fragments and $C_{i}$ is a column vector containing the coefficients that defines the NOCV $\psi_{\mathrm{i}}$ of Eq. 4 . It follows further [28-34] that the deformation density $\Delta \rho$ of Eq. 2 can be expressed in the NOCV representation as a sum of pairs of complementary eigenfunctions $\left(\psi_{-\mathrm{k}}, \psi_{\mathrm{k}}\right)$ corresponding to the eigenvalues $-v_{k}$ and $+v_{k}$ with the same absolute value but opposite signs:

$\Delta \rho(r)=\sum_{k=1}^{M / 2} v_{k}\left[-\psi_{-k}^{2}(r)+\psi_{k}^{2}(r)\right]=\sum_{k=1}^{M / 2} \Delta \rho_{k}(r)$.

Expression (6) is the most important for the interpretation of NOCV, as it defines the charge-flow channels decomposing the overall deformation density. Therefore, in the present study we will not discuss the orbitals themselves, but only the respective deformation density contributions, $\Delta \rho_{\mathrm{k}}$. Examples of orbitals and their interpretation can be found elsewhere [28-30].

In the combined ETS-NOCV scheme $[35,36]$ the orbital interaction term $\left(\Delta \mathrm{E}_{\mathrm{orb}}\right)$ is expressed in terms of NOCV's as

$\Delta E_{\text {orb }}=\sum_{k=1}^{M / 2} v_{k}\left[-F_{-k,-k}^{T S}+F_{k, k}^{T S}\right]$

where the diagonal Kohn-Sham matrix elements are defined over NOCV's with respect to the transition state (TS). The advantage of the expression in Eq. 7 for $\Delta \mathrm{E}_{\text {orb }}$ over that of Eq. 3 is that only a few complementary NOCV pairs normally contribute significantly to $\Delta \mathrm{E}_{\text {orb }}$. We see from the above Eqs. 6, 7 that for each complementary NOCV pair, representing one of the charge deformations $\Delta \rho_{\mathrm{k}}$, not only can visualize $\Delta \rho_{\mathrm{k}}$ but also provide the energy contributions to the bond energy from $\Delta \rho_{\mathrm{k}}$ [28-34].

The total bonding enthalpies $\left(D_{e}=-\Delta E_{\text {int }}\right)$ reported here do not include zero point energy additions, finite temperature contributions or basis set superposition error corrections. Our interest here has been to study the electronic nature of hydrogen bond formation through trends in the (electronic) bond enthalpy as revealed by our recently proposed ETS-NOCV procedure $[35,36]$.

\section{Results and discussion}

Let us start our discussion from a qualitative description of hydrogen bonding in $\beta$-dimer of oxalic acid (2), which is more stable (by $9.9 \mathrm{kcal} \mathrm{mol}^{-1}$ ) compared to dimer exhibiting $\alpha$-conformation (1), Table 1. It is clear from Fig. 2 that three deformation density contributions, $\Delta \rho_{\sigma}$, $\Delta \rho_{\pi}^{R A H B}$ and $\Delta \rho_{\mathrm{pol}}$, based on complementary NOCV's Eq. 6, describe the formation of two equivalent $\mathrm{O}-\mathrm{-HO}$ interactions in 2. The dominant component $\sigma(\mathrm{O}-\mathrm{HO})$ originates from the donation of electron density from the lone pair of oxygen into the empty $\rho_{H-O}^{*}$, characterizing 
Table 1 ETS-energy decomposition ${ }^{1}$ (in $\mathrm{kcal} \mathrm{mol}^{-1}$ ) of the hydrogen bonding in 1-4

\begin{tabular}{llllll}
\hline Systems $^{2}$ & $\Delta \mathrm{E}_{\text {Pauli }}$ & $\Delta \mathrm{E}_{\text {elstat }}$ & $\Delta \mathrm{E}_{\text {orb }}$ & $\Delta \mathrm{E}_{\text {dist }}$ & $\Delta \mathrm{E}_{\text {int }}$ \\
\hline $\mathbf{1}$ & 12.3 & -11.1 & -7.7 & 0.5 & -6.0 \\
$\mathbf{2}$ & 51.5 & -36.7 & -35.9 & 5.3 & -15.9 \\
$\mathbf{3}$ & 51.7 & -36.8 & -36.3 & 5.4 & -15.9 \\
$\mathbf{4}$ & 103.6 & -74.4 & -71.6 & 10.7 & -31.6 \\
\hline
\end{tabular}

${ }^{1} \Delta \mathrm{E}_{\text {int }}=\Delta \mathrm{E}_{\text {Pauli }}+\Delta \mathrm{E}_{\text {elstat }}+\Delta \mathrm{E}_{\text {dist }}+\Delta \mathrm{E}_{\text {orb }}$.

${ }^{2}$ The labeling corresponds to Fig. 1

anti-bonding orbital of $\mathrm{H}-\mathrm{O}$ bond in the oxalic acid monomer. In addition, density is shifted from hydrogen of $\mathrm{H}-\mathrm{O}$ group of one oxalic acid monomer to oxygen of the second fragment. The density deformation $\left(\Delta \rho_{\sigma}\right)$ in the $\sigma$ network of $\mathbf{2}$ gives rise to an orbital interaction stabilization of $\Delta E_{\text {orb }}^{\sigma}=-28.3 \mathrm{kcal} \mathrm{mol}^{-1}$. We note that the participation of $\sigma_{H O}^{*}$ in the hydrogen bonding leads to a stretch of the H-O bond by $0.06 \AA$, compared to non-bonded monomer. The second contribution to $\Delta E_{o r b}$, presented in Fig. 2, is $\Delta \rho_{\pi}^{R A H B}$, with the corresponding energy $\Delta E_{\pi}^{R A H B}=-3.5 \mathrm{kcal} \mathrm{mol}^{-1}$. It is evident from the contour of $\Delta \rho_{\pi}^{R A H B}$ that this contribution characterizes $\pi$ polarization, which apparently justifies assignment of oxalic acid to the group of resonance assisted hydrogen bonded (RAHB) systems. We observed that the density depletion in the $\sigma$-network gives rise to density buildup in the $\pi$-frame work and visa versa. More specifically, we noted a depletion of electron density from the $\pi$-type orbital on the oxygen atom of the $\mathrm{OH}$ group. It leads to a contraction (by $0.05 \AA$ ) of the HO-C bond. At the same time charge is donated to the carbonyl oxygen by electron transfer to the $\pi_{C O}^{*}$ orbital. The result is an elongation of the $\mathrm{CO}$ carbonyl bond by $0.02 \AA$. Finally, the last contribution, $\Delta \rho_{\text {pol }}$, with the corresponding energy by $-4.1 \mathrm{kcal} \mathrm{mol}^{-1}$, shows the density changes within each oxalic acid monomer (so called intra-fragment polarizations).

The second stabilizing contribution in $\mathbf{2}$ comes from the electrostatic attraction $\left(\Delta \mathrm{E}_{\text {elstat }}\right)$ between the oxalic acid monomers. It amounts to $-36.7 \mathrm{kcal} \mathrm{mol}^{-1}$. Such an interaction is due to the stabilizing interaction between the $\stackrel{\delta+}{H}-\stackrel{\delta-}{O}$ dipole of the HO bond in one monomer and the $\delta^{\prime}-\stackrel{\delta^{\prime}+}{C}$ dipole of the carbonyl group of the second oxalic acid fragment. Further stabilization comes from the fact that the electron density of one fragment only partially can shield the interaction of the nuclei on the same fragment from the electron density on the other fragment. The reduced shielding is due to inter-penetration of the two fragment densities. It should be noted that $\Delta \mathrm{E}_{\text {orb }}$ and $\Delta \mathrm{E}_{\text {elstat }}$ are of the same importance (in absolute value by $\sim 36 \mathrm{kcal} \mathrm{mol}^{-1}$ ). In addition, it is worth emphasizing that $\sigma(\mathrm{O}-\mathrm{HO})$ component $(78 \%$ of $\Delta$ Eorb) is roughly eight times as important as $\pi$-RAHB contribution $(9.8 \%$ of $\Delta \mathrm{E}_{\text {orb }}$ ). At the same time, the stabilizing effect (originating from $\Delta \mathrm{E}_{\text {orb }}$ and $\Delta \mathrm{E}_{\text {elstat }}$ ) is reduced by the repulsive interaction between the occupied carbonyl lone-pair orbital on one monomer and the occupied O-H bonding orbital on the other oxalic acid fragment, what is accordingly manifested by considerable Pauli repulsion contribution $\left(\Delta \mathrm{E}_{\text {Pauli }}\right)$, by $51.5 \mathrm{kcal} \mathrm{mol}^{-1}$, Table 1 . The total stabilization stemming from all bonding components Eq. 1, given per one hydrogen $\mathrm{O}-\mathrm{HO}$ bond, is by $\Delta \mathrm{E}_{\text {int }} / 2=-7.95 \mathrm{kcal} \mathrm{mol}^{-1}$,
Fig. 2 The contours of deformation density contributions $\Delta \rho_{\sigma}, \Delta \rho_{\pi}^{R A H B}, \Delta \rho_{\text {pol }}$ describing the hydrogen bonding between the monomers in 2 . In addition the corresponding ETS-NOCVbased energies (in kcal mol${ }^{-1}$ ) are shown. The numerically smallest contour values are \pm 0.001 a.u.

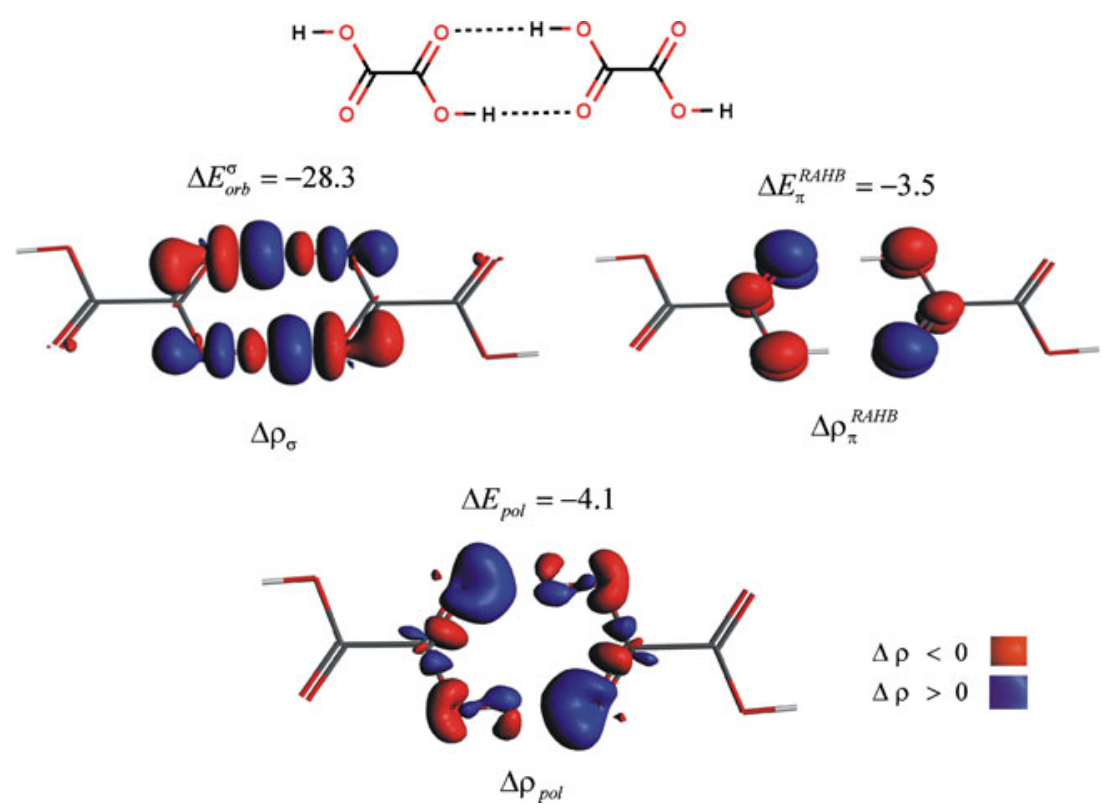


Fig. 3 The contours of deformation density contributions $\Delta \rho_{\sigma}, \Delta \rho_{\pi}^{R A H B}$ describing the hydrogen bonding between the monomers in $\mathbf{1}$. In addition the corresponding ETS-NOCVbased energies (in kcal mol ${ }^{-1}$ ) are shown. The numerically smallest contour values are \pm 0.001 a.u.

$$
\Delta E_{o r b}^{\sigma}=-5.6
$$

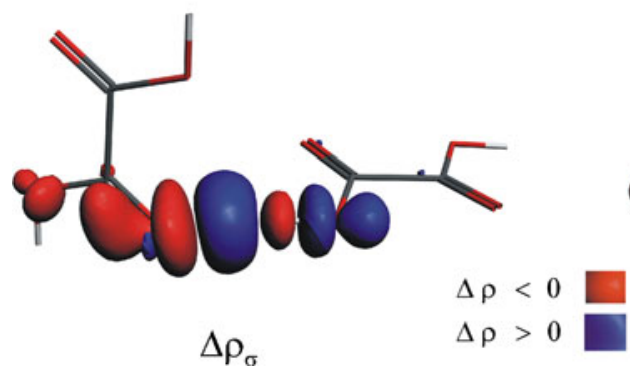

$\Delta E_{\pi}^{R A H B}=-0.7$

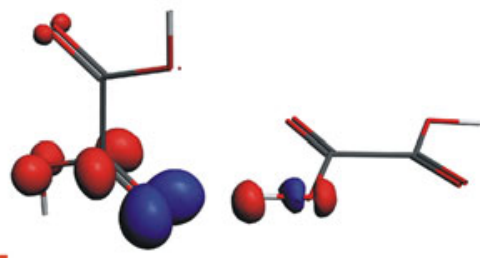

$\Delta \rho_{\pi}^{R A H B}$
Table 1. It is made up from the orbital interaction stabilization, $\Delta \mathrm{E}_{\text {orb }} / 2=-17.95 \mathrm{kcal} \mathrm{mol}^{-1}$ and destabilizing $\left(\Delta \mathrm{E}_{\text {elstat }}+\Delta \mathrm{E}_{\text {Pauli }}+\Delta \mathrm{E}_{\text {dist }}\right) / 2=10.0 \mathrm{kcal} \mathrm{mol}^{-1}$.

The ETS results gathered in Table 1 lead to the conclusion that $\alpha$-conformation of oxalic acid dimer (1) is energetically less stable than the corresponding $\beta$-structure $\left(\left|\Delta \mathrm{E}_{\text {int }}\right|\right.$ lower by $\left.9.9 \mathrm{kcal} \mathrm{mol}^{-1}\right)$, as a result of changes in all of the bonding contributions defined in Eq. 1. The main reason of a relatively small stabilization from $\Delta \mathrm{E}_{\text {orb }}$ is that solely one $\mathrm{O}-$-HO bond is formed between the oxalic acid monomers. The qualitative picture of $\sigma(\mathrm{O}-\mathrm{HO})$ formation (see Fig. 3) is analogous to $\mathbf{2}$, with practically no participation $\left(<1 \mathrm{kcal} \mathrm{mol}^{-1}\right)$ from the $\pi$-network. The lack of considerable RAHB contribution is related to the broken planarity in $\mathbf{1}$. The lower destabilization from Pauli term, as well as lower stabilization from electrostatic contribution for $\mathbf{1}$ originate from the fact that the monomers are further apart (by $0.2 \AA$ ), compared to $\beta$-dimer (2). We should remark at this point that the experimental lattice energy estimations for $\alpha$-and $\beta$-conformations of oxalic acid in the crystal show that these two forms are roughly similar in stability [55]. This is not consistent with our result. Apparently, the dimeric and trimeric structures are not appropriate as models of real, infinite, periodic crystals. However, it was not our intention. As it was stated in the Introduction section, the main goal of our investigation was to verify the applicability of our new ETS-NOCV scheme in a description of bonding in hydrogen bonded molecules that exhibit both $\sigma(\mathrm{O}-\mathrm{HO})$ and $\pi$ (RAHB) interactions. It would be worthy to conduct in the future a similar energy decomposition analysis (ETS), as implemented in the BAND program [56], which is suitable for periodic calculations. Such an analysis could shed light on the origin of stability for both oxalic acid conformations in the crystal [55].

It is obvious from three deformation density contributions and their corresponding energies, depicted in Fig. 4, that there is practically no difference in the picture of $\mathrm{O}$ $\mathrm{HO}$ bonding, neither qualitative nor quantitative when comparing to dimer 2 (Fig. 2). Furthermore, the data collected in Table 1 lead to the conclusion that not only electronic factor $\Delta \mathrm{E}_{\text {orb }}$, but as well the remaining contributions, i.e., Pauli repulsion, electrostatic and distorsion terms, are of the same magnitude, as in the case of $\mathbf{2}$. Accordingly, the total interaction energy, $\Delta \mathrm{E}_{\text {int }}$, in trimer $\mathbf{3}$ is exactly the same as for dimer $\mathbf{2}$. The same qualitative picture of bonding formation in $\mathbf{2}$ and $\mathbf{3}$ lead to the conclusion that NOCV's exhibit a feature of localized orbitals, i.e. they reveal the same characteristics of a given bond when going from one molecule to another.

Up to this point we have analyzed the two O--HO interactions in trimer 3, i.e., the trimer was divided up into
Fig. 4 The contours of deformation density contributions $\Delta \rho_{\sigma}, \Delta \rho_{\pi}^{R A H B}, \Delta \rho_{\text {pol }}$ describing the hydrogen bonding in $\mathbf{3}$. In addition the corresponding ETSNOCV-based energies (in $\mathrm{kcal} \mathrm{mol}{ }^{-1}$ ) are shown. The numerically smallest contour values are \pm 0.001 a.u.

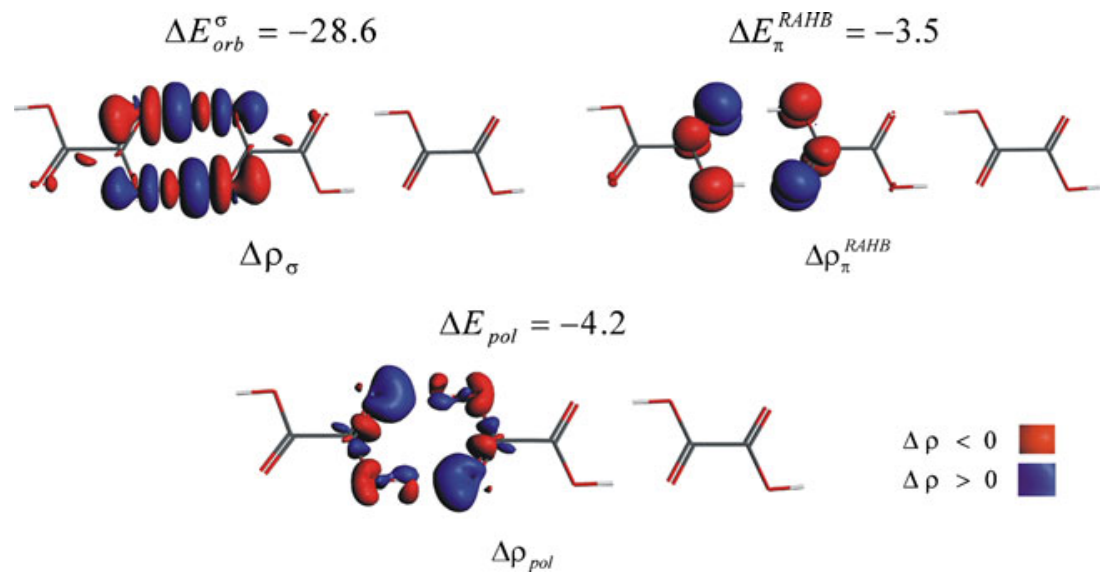


Fig. 5 The contours of deformation density contributions $\Delta \rho_{\sigma}, \Delta \rho_{\pi}^{R A H B}, \Delta \rho_{\text {pol }}$ describing the hydrogen bonding in 4 . In addition the corresponding ETSNOCV-based energies (in $\mathrm{kcal} \mathrm{mol}^{-1}$ ) are shown. The numerically smallest contour values are \pm 0.001 a.u.

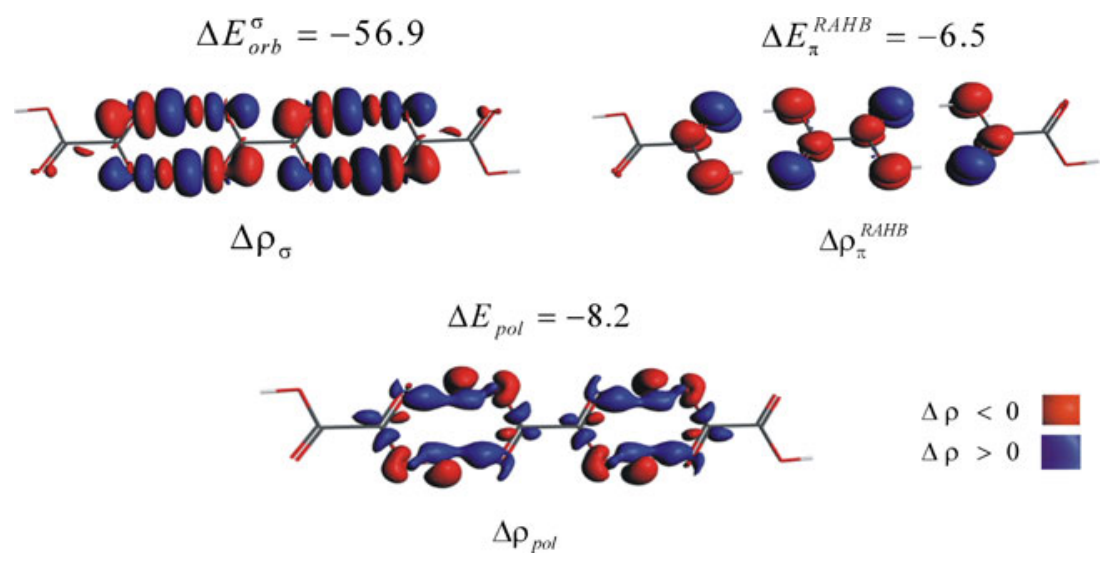

Thymine and Guanine-Cytosine base pairs that the dominant contribution comes from the $\sigma$-component, whereas $\pi$ contribution adds very little stabilization [46]. This is a similar trend to our findings for oxalic acid molecules.

\section{Concluding remarks}

In the present study we have characterized hydrogen bonding in the dimer and the trimer of $\alpha$-and $\beta$-oxalic acid, based on the recently proposed ETS-NOCV procedure. The main attention was paid to characterization of HB from both qualitative (by providing deformation density contributions, $\Delta \rho_{\mathrm{k}}$ ) and a quantitative (by providing energetic estimations $\Delta E_{k}$ for each $\Delta \rho_{\mathrm{k}}$ ) point of view.

We found based on the NOCV-deformation density contours that the formation of hydrogen bonding is associated with the electronic charge redistribution in both the $\sigma-\left(\Delta \rho_{\sigma}\right)$ and $\pi$-networks $\left(\Delta \rho_{\pi}\right)$. It was shown that $\sigma$ donation is realized by electron transfer from the lone pair of oxygen on one monomer into the empty $\rho_{H-O}^{*}$ orbital of the second oxalic acid fragment. In addition, a covalent contribution is observed by the density transfer from hydrogen of $\mathrm{H}-\mathrm{O}$ group in one oxalic acid monomer to the oxygen atom of the second fragment. The resonance assisted component $\left(\Delta \rho_{\pi}\right)$, RAHB, is based on the transfer of electron density from the $\pi$-orbital localized on the oxygen of $\mathrm{OH}$ on one oxalic acid monomer to the oxygen atom of the other fragment. The $\sigma(\mathrm{O}-\mathrm{HO})$ component is roughly eight times as important as $\pi$-contribution in $\beta$ oxalic acid dimer $(2)$ and trimers $(\mathbf{3 , 4})$. The electrostatic factor $\left(\Delta \mathrm{E}_{\text {elstat }}\right)$ is quantitatively as important as orbital interaction term $\left(\Delta \mathrm{E}_{\text {orb }}\right)$. Finally, comparing $\beta$-dimer (2) with trimer of oxalic acid (3) we found practically no difference in the characteristics of each of the $\mathrm{O}-\mathrm{HO}$ bonds, neither qualitative nor quantitative. 
Acknowledgments This work was supported by a research grant from the Polish Ministry of Science and Higher Education in Poland (N N204 227534).

Open Access This article is distributed under the terms of the Creative Commons Attribution Noncommercial License which permits any noncommercial use, distribution, and reproduction in any medium, provided the original author(s) and source are credited.

\section{References}

1. Jeffrey GA, Saenger W (1991) Hydrogen Bonding in Biological Structures. Springer, Berlin

2. Desiraju GR, Steiner T (1999) The Weak Hydrogen Bond in Structural Chemistry and Biology. Oxford University Press, New York

3. Lippincott ER, Schroeder R (1954) J Chem Phys 23:10991106

4. Reid C (1958) J Chem Phys 195830:182-190

5. Schneider WG (1955) J Chem Phys 23:26-30

6. Tsubomura H (1954) Bull Chem Soc Jpn 27:445-450

7. van Duijneveldt FB, Murrell JN (1967) J Chem Phys 46:17591767

8. Clementi E (1967) J Chem Phys 46:3851-3880

9. Mavri J (2007) J Chem Phys 126:205101-205109

10. Tuttle T, Grafenstein J, Wu A, Kraka E, Cremer D (2004) J Phys Chem B 108:1115-1129

11. Isaacs ED, Shukla A, Platzman PM, Hamann DR, Barbiellini B, Tulk CA (1999) Phys Rev Lett 82:600-603

12. Umeyama H, Morokuma K (1977) J Am Chem Soc 99:13161332

13. Morokuma K (1971) J Chem Phys 55:1236-1244

14. Panek JJ, Jezierska A (2007) J Phys Chem A 111:650-655

15. Jeziorski B, Moszynski R, Szalewicz K (1994) Chem Rev 94:1887-1930

16. Kaur D, Kohli R (2007) Int J Quantum Chem 108:119-134

17. Pakiari AH, Eskandari K (2006) J Mol Struct 759:51-60

18. Glendening ED (2005) J Phys Chem A 109:11936-11940

19. Pendás AM, Blanco MA, Francisco E (2006) J Chem Phys 125:184112-184120

20. Gilli P, Bertloasi V, Ferretti V, Gilli G (1994) J Am Chem Soc 116:909-915

21. Gilli G, Gilli P (2000) J Mol Struct 552:1-15

22. Kitaura K, Morokuma K (1976) Int J Quantum Chem 10:325-340

23. Stevens WJ, Fink WH (1987) Chem Phys Lett 139:15-22

24. Mo Y, Gao J, Peyerimhoff SD (2000) J Chem Phys 112:5530-5538

25. van der Vaart A, Merz KM (1999) J Phys Chem A 103:3321-3329

26. Ziegler T, Rauk A (1979) Inorg Chem 18:1755-1759

27. Ziegler T, Rauk A (1977) Theor Chim Acta 46:1-10

28. Mitoraj M, Michalak A (2007) J Mol Model 13:347-355
29. Mitoraj M, Michalak A (2007) Organometallics 26:6576-6580

30. Mitoraj M, Michalak A (2008) J Mol Model 14:681-687

31. Michalak A, Mitoraj M, Ziegler T (2008) J Phys Chem A 112:1933-1939

32. Mitoraj M, Zhu H, Michalak A, Ziegler T (2008) Int J Quantum Chem 109:3379-3386

33. Srebro M, Mitoraj M (2009) Organometallics 28:3650-3655

34. Srebro M, Michalak A (2009) Inorg Chem 48:5361-5369

35. Mitoraj M, Michalak A, Ziegler T (2009) J Chem Theory Comput 5:962-975

36. Mitoraj M, Michalak A, Ziegler T (2009) Organometallics 28:3727-3733

37. Allen FH (2002) Acta Cryst B 58:380-388

38. Allen FH, Motherwell WDS (2002) Acta Cryst B 58:407-422

39. te Velde G, Bickelhaupt FM, Baerends EJ, Fonseca Guerra C, Van Gisbergen SJA, Snijders JG, Ziegler T (2001) J Comput Chem 22:931-967, and refs therein

40. Baerends EJ, Ellis DE, Ros P (1973) Chem Phys 2:41-541

41. Baerends EJ, Ros P (1973) Chem Phys 2:52-59

42. te Velde G, Baerends EJ (1992) J Comput Phys 99:84-98

43. Fonesca Geurra C, Visser O, Snijders JG, te Velde G, Baerends EJ (1995) In: Clementi E, Corongiu G (eds) Methods and techniques in computational chemistry METACC-9. STEF, Cagliari, pp. 303395

44. Becke A (1988) Phys Rev A 38:3098-3100

45. Perdew JP (1986) Phys Rev B 34:7406-7406

46. Guerra CF, Bickelhaupt FM, Snijders JG, Baerends EJ (1999) Chem Eur J 5:3581-3594

47. ADF-GUI 2009.01, SCM, Amsterdam, The Netherlands, http:// www.scm.com (Visser O, Leyronnas P, van Zeist WJ, Lupki M)

48. Nalewajski RF, Mrozek J (1994) Int J Quantum Chem 51:187200

49. Nalewajski RF, Mrozek J, Formosinho SJ, Varandas AJC (1994) Int J Quantum Chem 52:1153-1176

50. Nalewajski RF, Mrozek J (1996) Int J Quantum Chem 57:377389

51. Nalewajski RF, Mrozek J, Mazur G (1996) Can J Chem 74:11211130

52. Nalewajski RF, Mrozek J, Michalak A (1997) Int J Quantum Chem 61:589-601

53. Nalewajski RF, Mrozek J, Michalak A (1998) Pol J Chem 72:1779-1791

54. Michalak A, De Kock R, Ziegler T (2008) J Phys Chem A $112: 7256-7263$

55. Raabe G (2002) Z Naturforsch 57a:961-966

56. BAND2009.01, SCM, Theoretical Chemistry, Vrije Universiteit, Amsterdam, The Netherlands, http://www.scm.com (te Velde G, Baerends EJ, Philipsen PHT, Wiesenekker G, Groeneveld JA, Berger JA, de Boeij PL, Klooster R, Kootstra F, Romaniello P, Snijders JG, Kadantsev ES, Ziegler T)

57. Góra RW, Grabowski SJ, Leszczyński J (2005) J Phys Chem A 109:6397-6405

58. Beck JF, Mo Y (2007) J Comput Chem 28:455-466 